\title{
Programa Educativo-Preventivo e a Higiene Bucal de Escolares: Estudo Clínico
}

\section{Educational-Preventive Program and Oral Hygiene of Schoolchildren: Clinical Study}

\author{
Suéllen Peixoto de Medeiros Urquiza ${ }^{1,2}$ \\ Monique Gonçalves D'Alessandro de Barros e Moraes ${ }^{1}$ \\ Maressa Borges dos Reis ${ }^{1,3}$ \\ Kenia Aparecida Freitas Moraes ${ }^{1,4}$ \\ Ana Flavia Calvo ${ }^{5}$ \\ Isabela Floriano ${ }^{5,6}$ \\ José Carlos Imparato ${ }^{5,7}$
}

\section{RESUMO}

Objetivo: O objetivo deste estudo foi avaliar a influência de atividades educativas e preventivas na higiene bucal de escolares. Metodologia: Foram avaliados 40 alunos de 6 a 8 anos, na Cidade de Patos-PB. Estes foram divididos em dois grupos: experimental e controle e foram examinados em relação às características socioeconômicas e de higiene bucal, assim como submetidos aos exames de ISG (Índice de sangramento gengival) e IHO-S (Índice de higiene oral simplificado). As crianças do grupo experimental participaram de um programa educativo-preventivo de 4 semanas, com orientações sobre a higiene bucal, os hábitos dietéticos e outras informações relevantes para a saúde bucal, além de realizarem, a cada sessão do programa, a escovação supervisionada. Quatro semanas após o exame clínico inicial, todas foram reavaliadas clinicamente, a fim de retomar os índices. Resultados: Os resultados mostraram que ocorreram diferenças estatisticamente significativas em relação ao ISG $(p<0,001)$ e atividade de gengivite $(p=0,008)$ para os dois grupos. Inicialmente, a mediana do ISG foi de $15,50 \%$, caindo para $8,00 \%$ após a realização das atividades. Além disso, o percentual de escolares com atividade de gengivite alta, que era na primeira avaliação de $65,0 \%$, caiu para $25,0 \%$, evidenciando uma influência positiva das atividades educativas e preventivas na higiene bucal dos escolares. Conclusão: Concluiu-se que um programa educativo-preventivo, em curto prazo, em nível escolar, é eficaz na redução da atividade de gengivite e na melhoria da higiene bucal. Não obstante, para que os resultados positivos possam se prolongar em longo prazo, o programa educativo-preventivo deverá ter caráter permanente.

\section{DESCRITORES}

Educação em Saúde. Motivação. Saúde Bucal.

\begin{abstract}
Objective: The aim of this study was to evaluate the influence of educational and preventive activities on the oral hygiene of schoolchildren. Methodology: 40 students aged 6 to 8 years were evaluated in Patos-PB. These were divided into two groups, experimental and control, and were examined for socioeconomic and oral hygiene characteristics, as well as subjected to ISG (Gingival Bleeding Index) and IHO-S (Simplified Oral Hygiene Index) exams. The children in the experimental group participated in a 4-week educational-preventive program, with guidance on oral hygiene, dietary habits and other information relevant to oral health, in addition to carrying out supervised brushing at each session of the program. Four weeks after the initial clinical examination, all children were reassessed clinically to resume the indexes. Results: The results showed that there were statistically significant differences in relation to the ISG $(p<0.001)$ and gingivitis activity $(p=0.008)$ for both groups. Initially, the median of the ISG was $15.50 \%$, falling to $8.00 \%$ after carrying out the activities. In addition, the percentage of students with high gingivitis activity, which was $65.0 \%$ in the first assessment, dropped to $25.0 \%$, signalling a positive influence of educational and preventive activities on the students' oral hygiene. Conclusion: It was concluded that a short-term educational-preventive program at school level is effective in reducing gingivitis activity and improving oral hygiene. However, for the positive results to be prolonged into the long term, the educational-preventive program must be permanent.
\end{abstract}

\section{DESCRIPTORS}

Health Education. Motivation. Oral Health.

${ }^{1}$ Discente da Pós-graduação em Odontopediatria, Faculdade São Leopoldo Mandic, Campinas, São Paulo, Brasil.

2 Professor Titular, Curso de Odontologia, Centro Universitário de Patos - UNIFIP, Patos, Paraíba, Brasil.

${ }^{3}$ Professor Assistente, Curso de Odontologia, Universidade Gurupi, Gurupi, Tocantins, Brasil.

${ }^{4}$ Professor Titular, Curso de Odontologia, Faculdade Pitágoras, Ipatinga, Minas Gerais, Brasil.

${ }^{5}$ Professor, Pós-Graduação em Odontopediatria, Faculdade São Leopoldo Mandic, Campinas, São Paulo, Brasil.

${ }^{6}$ Professor Titular, Curso de Odontologia, Centro Universitário Uninovafapi, Teresina, Piauí, Brasil.

${ }^{7}$ Professor Livre-Docente, Departamento de Ortodontia e Odontopediatria, Universidade de São Paulo, São Paulo, São Paulo, Brasil. 
$\mathrm{D}$ e acordo com a Organização Mundial de Saúde1, a saúde bucal é um indicador primordial da saúde geral, bem-estar e qualidade de vida. Estima-se que as doenças bucais afetam 3,5 bilhões de pessoas em todo o mundo, sendo a cárie dentária uma das doenças não transmissíveis mais prevalentes ${ }^{1}$. A situação epidemiológica brasileira ainda tem sido considerada grave, apresentando altos índices de prevalência de doenças bucais, em particular a cárie dentária e a doença periodontal ${ }^{2,3}$.

Tendo em vista o caráter sociocomportamental da doença cárie, é importante que sejam instituídas medidas educativo-preventivas, para que as pessoas tenham conhecimento sobre os fatores que afetam o processo saúde-doença e modifiquem positivamente os seus comportamentos ${ }^{4}$. No entanto, devem ser trabalhadas o mais precocemente possível para que as pessoas adquiram bons hábitos de saúde bucal5,6. A escola atua de maneira significativa na formação e na construção dos sujeitos, especialmente com relação aos hábitos $^{7}$. Sendo assim, muitos autores acreditam que este seja um local adequado para o desenvolvimento de programas de saúde envolvendo a população infantil, por reunir crianças em faixas etárias propícias à adoção de medidas educativas e preventivas ${ }^{8-10}$.

As atividades nos programas escolares incluem educação/promoção da saúde bucal, escovação supervisionada, aplicação de selantes de fóssulas e fissuras e/ou vários tratamentos. Essas atividades podem moldar as crenças, atitudes, valores e comportamentos relacionados à saúde das crianças. Além disso, as evidências sugerem que os serviços de saúde bucal na infância podem influenciar o estilo de vida saudável na idade adulta ${ }^{11,12}$.

A literatura aponta que não existe evidência suficiente sobre a eficácia das intervenções educativas na escola primária na redução da incidência de cárie ${ }^{13}$. Sendo assim, estudos clínicos que avaliem tais atividades devem ser realizados. O objetivo deste estudo foi avaliar o impacto de atividades educativo-preventivas na escola na higiene bucal de crianças.

\section{METODOLOGIA}

Desenho do estudo e critérios de elegibilidade

Trata-se de um estudo clínico randomizado, realizado no município de Patos-PB. A amostra por conveniência incluiu crianças de ambos os sexos, de 6 a 8 anos, regularmente matriculadas no $1^{\circ}$ e $2^{\circ}$ anos de uma escola municipal, de maio a julho de 2017. Foram excluídas as crianças que não estavam frequentando as aulas e/ou que estavam afastados por motivo de saúde; que fazem uso de aparelho ortodôntico; e/ou alunos que tinham déficit de habilidade psicomotora.

Sujeitos

As crianças foram alocadas aleatoriamente por sorteio em dois grupos: educativo $(E)$, que participou das atividades educativo-preventivas na escola, e controle (C), que não recebeu as referidas atividades durante o período de estudo. As crianças do grupo C foram contempladas com as atividades educativas ao final da coleta de dados. Não foram considerados o sexo e a condição de 
saúde bucal da criança para a composição dos grupos.

Coleta de dados

Os dados socioeconômicos, demográficos, de saúde geral e hábitos de saúde bucal das crianças foram obtidos por meio de um questionário estruturado, de acordo com Barreto et al. ${ }^{5}$. O questionário foi preenchido antes do exame clínico pelos pais ou responsáveis pela criança.

Os exames clínicos foram realizados antes e após a introdução do programa educativo-preventivo, no ambiente escolar, sob luz natural, estando a criança sentada em uma cadeira. Para realização dos exames, o examinador estava paramentado com os EPIs (equipamentos de proteção individual). Os índices foram registrados em uma ficha clínica, construída pelo próprio pesquisador.

Inicialmente, foi realizada a avaliação gengival com ISG - Índice de Sangramento Gengival. Dessa forma, a criança foi classificada com uma alta atividade de gengivite (acima de 10\%) ou uma baixa atividade de gengivite (até 10\%). Em seguida, foi obtido o IHO-S - Índice de Higiene Oral Simplificado.

\section{Intervenções}

Após o exame clínico inicial, o programa educativo-preventivo foi realizado junto às crianças designadas para o grupo experimental, constituindo-se de quatro palestras educativas semanais. Estas utilizaram recursos como o teatro de fantoches, as brincadeiras interativas, os macromodelos, escova e creme dental e diapositivos ilustrativos. O tempo médio em cada atividade foi de 40 minutos. Nestas atividades, foram enfatizados os seguintes temas: cárie dentária, gengivite, escovação com dentifrício fluoretado, uso de fio dental e relação da dieta com a cárie dentária. Utilizou-se uma linguagem clara, a fim de tornar a atividade atrativa para as crianças, motivando-as a adquirir novos hábitos de saúde bucal.

Após cada palestra, as crianças foram organizadas em grupos de 4 e realizou-se uma escovação supervisionada com dentifrício fluoretado 1100ppm. Todas elas receberam um kit com escova dental e dentifrício.

Quatro semanas após o exame clínico inicial, todas as crianças foram reavaliadas clinicamente, obedecendo à mesma sequência do exame inicial.

\section{Aspectos éticos}

Este estudo foi aprovado pelo Comitê de Ética em Pesquisa do Centro Universitário de Patos sob o Parecer $n^{\circ}$ 2.000.053.

\section{Análise Estatística}

Os dados coletados foram tabulados e submetidos à análise estatística descritiva. Foram calculadas as frequências absolutas e percentuais para as variáveis categóricas, bem como as medidas de tendência central e de variabilidade para as variáveis quantitativas. Em seguida, para avaliar a influência de atividades realizadas na higiene bucal dos escolares, foram empregados os testes de Wilcoxon para amostras pareadas (variáveis quantitativas com distribuição não normal avaliada pelo teste de Shapiro-Wilk), de McNemar 
(variáveis qualitativas nominais dicotômicas) e de homogeneidade marginal (variáveis qualitativas ordinais). O nível de significância foi fixado em $p<0,05$. Todas as análises foram conduzidas usando o software SPSS Statistics versão 20.0, considerando um intervalo de confiança de $95 \%$.

\section{RESULTADOS}

Participaram do estudo 40 crianças. A Tabela 1 mostra a distribuição da amostra de acordo com as características sociodemográficas das crianças. A maioria das crianças era do sexo feminino e tinha 8 anos de idade. A

Tabela 1. Características sociodemográficas das crianças

\begin{tabular}{|c|c|c|c|}
\hline \multirow{3}{*}{ Variáveis } & \multicolumn{2}{|c|}{ Grupo } & \multirow{3}{*}{\begin{tabular}{|c|} 
Total \\
$\mathrm{n}(\%)$
\end{tabular}} \\
\hline & Experimental & Controle & \\
\hline & $\mathrm{n}(\%)$ & $\mathrm{n}(\%)$ & \\
\hline \multicolumn{4}{|l|}{ Sexo } \\
\hline Feminino & $13(65,0)$ & $09(45,0)$ & $22(55,0)$ \\
\hline Masculino & $07(35,0)$ & $11(55,0)$ & $18(45,0)$ \\
\hline \multicolumn{4}{|l|}{ Filhos (anos) } \\
\hline 6 & $05(25,0)$ & $07(35,0)$ & $12(30,0)$ \\
\hline 7 & $03(15,0)$ & $06(30,0)$ & $09(22,5)$ \\
\hline 8 & $12(60,0)$ & $07(35,0)$ & $19(47,5)$ \\
\hline \multicolumn{4}{|l|}{ Questionário aplicado aos pais } \\
\hline \multicolumn{4}{|c|}{ Nível de escolaridade do responsável pela criança } \\
\hline Não estudou & - & $003(15,0)$ & $03(7,5)$ \\
\hline $1^{\circ}$ grau completo & $07(35,0)$ & $08(40,0)$ & $15(37,5)$ \\
\hline $2^{\circ}$ grau completo & $10(50,0)$ & $06(30,0)$ & $16(40,0)$ \\
\hline Superior completo & $03(15,0)$ & $03(15,0)$ & $06(15,0)$ \\
\hline \multicolumn{4}{|l|}{ Números de filhos } \\
\hline 1 & $03(15,0)$ & $02(10,0)$ & $05(12,5)$ \\
\hline$>1$ & $17(85,0)$ & $18(90,0)$ & $35(87,5)$ \\
\hline \multicolumn{4}{|l|}{ Ida anterior da criança ao dentista } \\
\hline Sim & $16(80,0)$ & $12(60,0)$ & $28(70,0)$ \\
\hline Não & $04(20,0)$ & $08(40,0)$ & $12(30,0)$ \\
\hline \multicolumn{4}{|c|}{ Responsável por realizar a escovação da criança } \\
\hline Criança & $16(80,0)$ & $16(80,0)$ & $32(80,0)$ \\
\hline Cuidador & $04(20,0)$ & $04(20,0)$ & $08(20,0)$ \\
\hline \multicolumn{4}{|c|}{ Frequência de escovação da criança } \\
\hline Até duas vezes ao dia & $13(65,0)$ & $10(50,0)$ & $23(57,5)$ \\
\hline Acima de duas vezes ao dia & $07(35,0)$ & $10(50,0)$ & $17(42,5)$ \\
\hline \multicolumn{4}{|c|}{ Idade em que o filho iniciou a escovação com creme dental } \\
\hline Antes de 1 ano de idade & $08(40,0)$ & $07(35,0)$ & $15(37,5)$ \\
\hline A partir de 1 ano de idade & $12(60,0)$ & $13(65,0)$ & $25(62,5)$ \\
\hline
\end{tabular}


maior parte dos responsáveis estudou até o $2^{\circ}$ grau e possuía mais de 1 filho. Cerca de $30 \%$ da amostra nunca tinha ido ao dentista.

A Tabela 2 mostra a higiene bucal dos escolares do grupo experimental no momento de avaliação inicial (T0) e final (T1). Diferenças foram observadas em relação ao ISG $(p<0,001)$ e atividade de gengivite $(p=$ $0,008)$. Inicialmente, a mediana do ISG foi de $15,50 \%$, caindo para $8,00 \%$ após a realização das atividades. Além disso, o percentual de escolares com atividade de gengivite alta que era na primeira avaliação de $65,0 \%(n=13)$, caiu para $25,0 \%(n=5)$.

A Tabela 3 mostra os resultados da análise comparativa da higiene bucal dos escolares pertencentes ao grupo controle no momento de avaliação inicial (T0) e final (T1). Não foram constatadas diferenças $(p>0,05)$.

\section{DISCUSSÃO}

Atividades educativas e preventivas fazem parte da rotina de escolares em alguns países. A aplicação de atividades educativo-preventivas para saúde bucal na escola, além de ser simples, de fácil aplicação e baixo custo é de grande valor na prevenção de doenças bucais causadas por biofilme $e^{1,14}$.

O presente estudo mostrou que o programa educativo-preventivo teve influência positiva nas condições de higiene bucal dos escolares. Resultados semelhantes foram achados em outros estudos $5,7,15$.

Informações sobre a saúde bucal associada às ações preventivas tais como a escovação supervisionada e a aplicação tópica de flúor, são eficazes na redução da placa visível e no sangramento gengival e, em longo prazo, geram uma redução na experiência de cárie ${ }^{5}$. Sendo assim, a orientação em saúde bucal deve ser implementada nos primeiros anos de vida, visto que nesta fase as crianças são mais receptivas e aprendem com mais facilidade, tendo maior possibilidade de aplicar essas orientações aos seus hábitos diários ${ }^{15}$.

Tabela 2. Análise comparativa da higiene bucal dos escolares pertencentes ao grupo experimental no momento de avaliação inicial (T0) e final (T1)

\begin{tabular}{|c|c|c|c|c|c|}
\hline \multirow[t]{2}{*}{ Variáveis } & \multicolumn{4}{|c|}{ Situação Avaliada } & \\
\hline & TO & & & p-valor & \\
\hline \multicolumn{6}{|l|}{ ISG } \\
\hline Mediana (intervalo interquartil) & \multicolumn{2}{|c|}{$15,50(13,00)$} & \multicolumn{2}{|c|}{$8,00(7,00)$} & $<0,001^{(\mathrm{a}) *}$ \\
\hline Categorias & $\mathrm{n}$ & $\%$ & $\mathrm{n}$ & $\%$ & \\
\hline Atividade de gengivite & & & & & $0,008^{(b)^{*}}$ \\
\hline Baixa & 07 & 35,0 & 15 & 75,0 & \\
\hline Alta & 13 & 65,0 & 05 & 25,0 & \\
\hline \multicolumn{6}{|l|}{ IHO-S } \\
\hline Dente limpo e total ausência de biofilme bacteriano & 13 & 65,0 & 17 & 85,0 & $0,102^{(c)}$ \\
\hline $\begin{array}{l}\text { Dente apresenta } 1 / 3 \text { de sua superfície com biofilme } \\
\text { bacteriano }\end{array}$ & 07 & 35,0 & 03 & 15,0 & \\
\hline
\end{tabular}


Tabela 3. Análise comparativa da higiene bucal dos escolares pertencentes ao grupo controle no momento de avaliação inicial (T0) e final (T1)

\begin{tabular}{|c|c|c|c|c|c|}
\hline \multirow{2}{*}{ Variáveis } & \multicolumn{4}{|c|}{ Situação Avaliada } & \multirow[b]{2}{*}{ p-valor } \\
\hline & \multicolumn{2}{|c|}{ TO } & \multicolumn{2}{|c|}{$\mathrm{T} 1$} & \\
\hline \multicolumn{6}{|l|}{ ISG } \\
\hline Mediana (intervalo interquartil) & \multicolumn{2}{|c|}{$4,00(11,00)$} & \multicolumn{2}{|c|}{$5,50(14,00)$} & $0,154^{(a)}$ \\
\hline Categorias & $\mathrm{n}$ & $\%$ & $\mathrm{n}$ & $\%$ & \\
\hline Atividade de gengivite & & & & & $1,000^{(b)}$ \\
\hline Baixa & 14 & 70,0 & 13 & 65,0 & \\
\hline Alta & 06 & 30,0 & 07 & 35,0 & \\
\hline \multicolumn{6}{|l|}{ IHO-S } \\
\hline $\begin{array}{l}\text { Dente limpo e total ausência de biofilme } \\
\text { bacteriano }\end{array}$ & 03 & 15,0 & 02 & 10,0 & $0,739^{(\mathrm{c})}$ \\
\hline $\begin{array}{l}\text { Dente apresenta } 1 / 3 \text { de sua superfície com } \\
\text { biofilme bacteriano }\end{array}$ & 05 & 25,0 & 06 & 30,0 & \\
\hline $\begin{array}{l}\text { Dente apresenta biofilme até } 1 / 2 \text { da sua } \\
\text { superfície }\end{array}$ & 09 & 45,0 & 11 & 55,0 & \\
\hline $\begin{array}{l}\text { Dente apresenta biofilme além da } 1 / 2 \text { da } \\
\text { sua superfície }\end{array}$ & 03 & 15,0 & 01 & 5,0 & \\
\hline
\end{tabular}

Nota. ${ }^{\text {(a) }}$ Teste de Wilcoxon para amostras pareadas; ${ }^{(b)}$ Teste de McNemar; ${ }^{(c)}$ Teste de Homogeneidade Marginal.

As abordagens e medidas educativo-preventivas em nível escolar tem superioridade em relação as abordagens individuais em consultórios e as estratégias em escolas são uma maneira moderna de prevenção odontológica. As orientações de higiene bucal podem ser realizadas por qualquer pessoa que tenha conhecimentos sobre saúde bucal e fatores de risco ${ }^{16}$. Em vários países ao redor do mundo, as crianças são orientadas a escovar diariamente (na pré-escola e escola) com dentifrício fluoretado, sendo a quantidade de acordo com a faixa etária da criança, orientando-as para não o deglutir ${ }^{1}$. Estados e municípios devem estabelecer programas de educação em saúde bucal em pré-escolas, incluindo programas de escovação com dentifrício fluoretado (de 1000-1500 ppm), sendo eficaz em reduzir a cárie dentária quando as atividades são realizadas por professores capacitados $^{17,18}$.

É importante ressaltar que os programas educativo-preventivos estabelecidos sejam regulares e que as crianças participantes sejam constantemente reavaliadas, visando o sucesso mudança de comportamentos e consequente redução de doenças ${ }^{9}$. Uma revisão sistemática e meta-análise realizada em 2017 com o objetivo de determinar a eficácia dos programas de educação e promoção em saúde bucal concluiu que estes são eficazes e têm impactos positivos relacionados às consultas odontológicas, atitudes, bem como hábitos de escovação e uso do fio dental durante 3 meses pós-intervenção ${ }^{19}$. Assim, existe ainda a necessidade de avaliá-los me- 
Ihor e em um maior espaço de tempo da sua aplicabilidade ${ }^{3}$. Intervenções educativas com no mínimo quatro contatos com o público-alvo apresentam maiores chances de sucesso ${ }^{20}$.

De acordo com os resultados constatados neste estudo, evidenciou-se a importância da coleta inicial dos índices de higiene oral do paciente e o sangramento gengival, antes de iniciar a realização de programas educativo-preventivos. Ainda, periodicamente, estes índices devem ser retomados, de forma a monitorar se as ações realizadas têm sido efetivas na prevenção de doenças bucais. Caso as ações não estejam cumprindo o seu papel, é preciso que sejam revistas e replanejadas, a fim de alcançar os objetivos propostos, que seja a melhoria das condições de saúde bucal. Vale salientar que reforços motivacionais devem ser realizados no decorrer das ações, para tornar os resultados duradouros.

\section{CONCLUSÃO}

Conclui-se que a atividade educativo-preventiva empregada teve impacto positivo na higiene bucal das crianças e reduziu os índices de sangramento gengival e de acúmulo de biofilme.

\section{REFERÊNCIAS}

1. Organização Mundial da Saúde Ending Childhood Dental Caries. Manual de implementação da OMS. Programa de Saúde Bucal, Prevenção de Doenças Não Transmissíveis. Sede da OMS (2019).

2. Gasparinio LCS. Educação em saúde bucal: o uso de ferramentas lúdicas na escola. [Trabalho de Conclusão de Curso de Especialização em Estratégia Saúde da Família]. Formiga-MG: Universidade Federal de Minas Gerais; 2014. 29p.

3. Vilas Boas, PD. Impacto de programas educativos sobre condições bucais de escolares de 6 e 7 anos em duas escolas municipais do interior do Estado de São Paulo/ Brasil. [Monografia de Odontologia]. Piracicaba: Universidade Estadual de Campinas; 2012. 40p.

4. Menegaz AM, Silva AER,Cascaes AM. Intervenções educativas em serviços de saúde e saúde bucal: revisão sistemática. Rev Saúde Pública. 2018; 52:52.

5. Barreto DM, Paiva SM, Ramos-Jorge ML, Ferreira, MC Avaliação da eficácia de uma atividade educativo-preventiva com pré-escolares: estudo piloto de um ensaio clínico randomizado. Arq Odontol. 2013; 49 (3):113-121.

6. Souza MEM, Carvalho ÉS, Fernandes ABSP, Carvalho FK, Carvalhal CO, Dutra ALT. A educação em saúde como medida de prevenção e promoção da saúde bucal: Health education as a measure of prevention and promotion of oral health. Full Dentistry in Science. 2015; 6(22):239-248.

7. Silveira JLGC, Oliveira V, Padilha WWN. Avaliação da redução do índice de placa visível e do índice de sangramento gengival em uma prática de promoção de saúde bucal com crianças. Pesqui Odontol Bras. 2002; 16(2):169-174

8. Lemkuhl I, Souza MVC, Cascaes AM, Bastos JL. A efetividade das intervenções educativas em saúde bucal: revisão de literatura. Cad. Saúde Colet. 2015; 23(3):336346.

9. Alcantara TL, Batista, MJ, Gibilini C, Ferreira NP, Rosário de Sousa ML. Fatores associados à saúde bucal de pré-escolares inseridos em programa educativo preventivo no município de Piracicaba/SP. RPG Rev Pós Grad. 2011; 18(2):102-107.

10. Santos MO, Casotti CA, Queiroz APDG Carneiro JAO, Uemura TF. Conhecimento e percepção sobre saúde bucal de professores e pré-escolares de um município baiano. RFO. 2015, 20(2):172-178.

11. Alsumait A, EISalhy M, Amin M. Efeitos a longo prazo do programa de saúde bucal escolar nos conhecimentos e práticas de saúde bucal e na qualidade de vida relacionada à saúde bucal Med Princ Pract. 2015; 24(4):362-368.

12. Brownson RC, Haire-Joshu D, Luke DA-Moldando o contexto da saúde: uma revisão das abordagens ambientais e políticas na prevenção de doenças crônicas. Annu Rev Saúde Pública. 2006; 27: 341-370.

13. Cooper AM, O'Malley LA, Elison SN, Armstrong R, Burnside G, Adair P, Dugdill L, Pine C. Primary school-based behavioural interventions for preventing caries. Cochrane Database Syst Rev. 2013; 31(5):1-47.

14. Sityá DS, Giacomini $O$, Sangioni LA, Sendtko CRS, Unfer B. Análise de programas escolares de saúde bucal no Brasil. RFO. 2014; 19(3):293-296.

15- Santos NML, Maders C, Lemos VMA, Ziembowicz LFB. O Impacto do Programa preventivo-educativo da Unidade de 
Saúde da Família Viçosa na Saúde Bucal de Escolares. Rev. Fac. Odontol. 2010; 50(2):15-19.

16- Euba A, Paschos E, Mattner B, Storr U. Motivation zur Prävention im Kindesalter als Basis füreine nachhaltige Zahngesundheit - Das Augsburger Model /Motivation for Prevention in Childhood as a Basis for long-Term DentalHealth: The Augsburg Model. Gesundheitswesen. 2016; 78:103-106.

17. Jurgensen N, Petersen, PE. Promoting oral health of children through schools: results from a WHO global survey 2012. Community Dent Health. 2013; 30(4):204-218.

18. Petersen PE, Hunsrisakhun J, Thearmontree A, Pithpornchaiyakul S, Hintao J, Jurgensen N, et al. Schoolbased intervention for improving the oral health of children in southern Thailand. Community Dent Health. 2015; 32(1):44-50.
19- Ghaffari M, Rakhshanderou S, Ramzankhani A, Noroozi M, Armoon B. Oral health education and promotion programmes: Meta-analysis of 17-year intervention. Int J Dent Hygiene. 2018; 16:59-67.

20- Menegaz AM, Silva AER,Cascaes AM. Intervenções educativas em serviços de saúde e saúde bucal: revisão sistemática. Rev Saúde Pública. 2018; 52:52.

\section{CORRESPONDÊNCIA}

Suéllen Peixoto de Medeiros Urquiza

Rua Arnaldo Assis de Medeiros, 665, Novo Horizonte, 58704760, Patos/PB

E-mail: suellenpeixoto@hotmail.com 